\title{
Associations of physical activity with cardiorespiratory fitness, muscle strength, and body composition
}

Związek pomiędzy aktywnością fizyczną a wydolnością sercowo-naczyniową, siłą mięśni i składem ciała

\author{
'Samira Davarzani, ${ }^{1}$ Nadia Babaei, ${ }^{1}$ Mojdeh Ebaditabar, ${ }^{2}$ Kurosh Djafarian, ${ }^{1}$ Sakineh Shab-Bidar \\ ${ }^{1}$ Department of Community Nutrition, School of Nutritional Sciences and Dietetics, Tehran University \\ of Medical Sciences (TUMS), Tehran, Iran \\ ${ }^{2}$ Department of Clinical Nutrition, School of Nutritional Sciences and Dietetics, Tehran University \\ of Medical Sciences (TUMS), Tehran, Iran
}

\begin{abstract}
Introduction: Most studies have indicated the association between physical activity level (PAL) and physical fitness indices in children and adolescents. This study aimed to investigate the association of PAL with cardiorespiratory fitness, muscle strength, and body composition in healthy Iranian adults.

Method: This cross-sectional study was carried out on 120 males and 152 females aged 18-70 years. The participants underwent physical fitness measurements including grip strength, maximal oxygen consumption, and body composition. The PAL was objectively assessed using the International Physical Activity Questionnaire (IPAQ).

Results: We observed a significant association between PAL and body composition measurements $(p<0.05)$. Controlling for covariates did not change the results except for body mass index and waist circumference. All cardiorespiratory fitness and muscle strength indexes were positively associated with PAL $(p<0.001$ and $p=0.001$, respectively). However, these associations were disappeared after adjustment for age, gender, and BMI.

Conclusions: Improved body composition profile and function of the muscular and cardiovascular system were associated with higher PA. Further longitudinal studies are required to understand the causal direction of the relationship.

Key words:

body composition, physical activity, cardiorespiratory fitness, muscle strength.
\end{abstract}




\section{Introduction}

Physical activity (PA) is defined as any movement (not just exercise) that leads to energy expenditure by the musculoskeletal system [1]. Six percent of global deaths have resulted from insufficient PA [1, 2]. Physical activity promotes individual and public health in all ages [3]. Physical inactivity leads to an increased risk of obesity, insulin resistance, depression, cardiovascular diseases, fall-related injuries, and osteoporosis [4-6]. In contrast, elevated physical activity level (PAL) was associated with physical fitness. Physical fitness as one of the important markers of health consists of cardiorespiratory fitness, muscle strength, and body composition [7]. Poor cardiorespiratory fitness (CRF) and muscular strength are strong predictors of health problems and mortality [8, 9]. CRF has been defined as a capability of the circulatory and respiratory systems to carry oxygen to the muscles during PA and is evaluated by maximal oxygen uptake $\left(\mathrm{VO}_{2} \max \right)$ [10]. The amount of force that is produced by skeletal muscle has been considered as muscle strength $[11,12]$. Handgrip strength typically is an indicator of overall body strength [13]. Adequate PA particularly moderate to vigorous-intensity physical activity (MVPA) may lead to optimization of body composition including weight, low body fat mass, promotion bone, and muscle mass [2, 14, 15]. Moreover, muscle strength and CRF are reported to be directly associated with PAL $[2,14]$.

Most studies examined PA with muscle strength, CRF, and body composition individually. But a few studies assessed these associations comprehensively. Previous studies have demonstrated a positive relationship between fitness tests especially aerobic capacity and PA in children [16, 17]. Also, some reports have revealed that higher PAL leads to enhanced muscle strength in older adults [18]. Church et al. [19] found effective changes in fitness due to different levels of physical activity and reported that even a low level of physical activity leads to positive changes in postmenopausal women. Numerous studies have reported that PA has low to moderate relationships to physical fitness and health outcomes [20-22]. So far, most of the published studies have addressed the relation of PA to physical fitness in children and adolescents [23]. Therefore, the present study aimed to examine the association of PAL with cardiorespiratory fitness, muscle strength, and body composition in healthy Iranian adults.

\section{Material and methods}

\section{Study design and participants}

This cross-sectional study was performed from May to September 2018 in Tehran, Iran and 272 volunteers aged between 18 and 70 years were recruited. The sample size was calculated based on the previous articles $[24,25]$ by using the following equation ( $\alpha=5 \%, 1-\beta=95 \%$ ), the required sample size was equal to 136 people. To increase the accuracy and statistical power of the study, the sample size was doubled and enhanced to 272 people.

$$
\mathrm{n}=\left(\frac{\mathrm{z}_{1-\frac{\alpha}{2}}+\mathrm{z}_{1-\beta} \times \sqrt{1-r^{2}}}{r}\right)^{2}+3
$$

The exclusion criteria include: a) any treatment with androgenic supplements in last 12 month and anti-osteoporotic drugs, b) clinical and subclinical disease such as inflammatory diseases, arthritis, cardiovascular diseases (CVDs), chronic kidney disease (CKD), atopy, disability, asthma, pulmonary and endocrine disorders affecting muscle metabolism, c) pregnant and lactating women and d) inability to perform tests. Data on physical activity, demographic data such as age, sex, marital status, smoking status, blood pressure, and physical fitness tests were collected. All procedures were performed the ethical standards of the Tehran University of Medical Sciences (ethic code: IR.TUMS.VCR. REC.1397.472). All participants signed written consent forms.

\section{Anthropometric assessments and body composition}

Anthropometric data including height, weight, and waist circumference were recorded. Height was measured with a calibrated tape line fastened to a wall. Body-weight was assessed using digital scales with light clothes and without shoes. BMI, fat-free mass (FFM), fat mass (FM), waist circumference (WC), waist-hip ratio (WHR), body mass content (BMC), and clinical assessments were measured using a body composition analyzer (InBody Sweden, 2017). Conditions were carefully standardized as follows: no food intake for at least 4 hours, minimal drink of 2 I of water last day, inactivity at least $8 \mathrm{~h}$, no coffee or alcoholic beverage consumption during for at least 12 hours, and no diuretic use at least 24 hours [26].

\section{Blood pressure}

After 15 min of initial resting, blood pressure was determined twice with at least 30 seconds pause by a digital instrument (Beurer BP equipment, BC 08, digital, Germany). The average of 2 measurements was reported.

\section{Physical fitness tests}

CRF and handgrip strength (HGS) were assessed using a graded exercise treadmill and handgrip digital dynamometer, respectively. Participants were invited to a familiarization session with the devices and the test protocol was explained. Muscle health and range of motion of the joints were controlled by the trained person. People with severe symptomatic aortic stenosis, symptomatic heart failure without control, acute pulmonary embolism or pulmonary infarction, acute myocarditis or acute pericarditis, etc. were excluded from the study. Warming up was done for 5 minutes prior to starting the muscular fitness test. The subjects began to exercise at an easy speed (8-10 km per $1 \mathrm{~h}$ ) and without incline on a graded treadmill.

\section{Muscular strength}

Maximum grip strength was measured using a digital handgrip dynamometer (Saehan, model SH5003; Saehan Corporation, Masan, South Korea) [27]. Subjects were asked to sit on a chair, the shoulder was rotated naturally, the elbow was flexed 
Związek pomiędzy aktywnością fizyczną a wydolnością sercowo-naczyniową,

to 90 degrees from a natural position, and the forearm and wrist in a normal position. then the participants squeezed the dynamometer with maximum isometric effort. The test was performed three times for right and left hands. The average of three efforts in both hands was considered in data analyses.

\section{CRF}

CRF was determined by a maximal graded exercise test following the Bruce protocol [28]. Maximum oxygen consumption is the common laboratory marker for CRF, which was measured by a treadmill and respiratory gas analyzer (Cortex Metalesizer3B). Treadmill exercise has been designed in seven steps with elevation in speed and incline at three-minute intervals for each step, while the gas analyzer mask was on the subjects' faces. In the first step, the subject began walking at $2.74 \mathrm{~km} / \mathrm{h}$ with an incline equal to $10 \%$. In each step, the speed of the treadmill has been increased and the incline was increased by $2 \%$ until the seventh step of this protocol or until the subject was exhausted. According to the Bruce protocol, the exercise could be terminated if the patient's heart rate reached above $90 \%$ of the maximum heart rate, respiratory exchange ratio went up to 1.1, or voluntary ending of exercise test by the participants.

\section{Physical activity}

We used the International Physical Activity Questionnaire (IPAQ) to assess the physical activity of the participants during the last week [29]. The validity and reliability of the Iranian version of this questionnaire have been confirmed [30]. In addition to leisure-time PA as a hobby, activities such as transportation, work, and housekeeping are also considered, which have led to increasing evaluation accuracy and significant association with health outcomes. According to the IPAQ criteria, data were recorded regarding vigorous and moderate activity and walking during the previous 7 days. Duration and frequency of activity days were multiplied by the metabolic equivalent task value of the activity to calculate the total activity. The total physical activity per week was categorized into three groups (low, moderate, and high) according to the IPAQ scoring protocol [29]. Individuals who meet each of the following criteria are considered moderate. a) three or more days of high-intensity activity for at least 20 minutes per day $\mathrm{OR}$ b) five or more days of walking or moderate activity for at least 30 minutes per day OR c) five or more days walking, moderate or high-intensity activities reaching at least 600 MET-minutes per week. Subjects who meet each of the following criteria are considered high: a) three or more days of vigorous-intensity activity and achieving at least 1500 MET-minutes per week OR b) seven days of any combination of activities such as walking, moderate or vigorous intensity with at least 3000 MET minutes per week. Those subjects are not classified in category 2 or 3 are considered low. Also, IPAQ was computed for a continuous score and reported as metabolic equivalent (MET)-minutes per week.

\section{Statistical analysis}

Descriptive information is displayed as mean (SD) or frequency (percentages). Cardiorespiratory fitness is expressed in $\mathrm{l} / \mathrm{min}, \mathrm{ml} / \mathrm{min} / \mathrm{kg}$ BW and $\mathrm{ml} / \mathrm{min} / \mathrm{kg}$ LBM and muscle strength are presented by right and left handgrip $(\mathrm{kg})$, an average of right and left handgrip are considered as $\mathrm{kg}$ and $\mathrm{kg} / \mathrm{kg} \mathrm{BW}$. At first, unadjusted associations were investigated using ANOVA. Subsequently, ANCOVA was performed for adjusted models. Age, sex, and smoking were adjusted due to the effect on body composition. Additionally, models that investigate relationships between PAL and physical fitness tests were adjusted for age, sex, and BMI. Also, linear regression was done to investigate the associations between MET (continuous score of PA) as independent variables and some factors of body composition, HGS, and $\mathrm{VO}_{2}$ peak. All statistical tests were stratified by PAL and analyzed using SPSS Statistics version 25.0. (IBM Corporation, USA).

\section{Results}

The characteristics of the participants are shown in Table I. Mean (SD) for age and BMI were 37 (13) and 25.6 (4.6), respectively.

Table I. Characteristics, anthropometric, body composition, and physical fitness measurements

\begin{tabular}{|c|c|c|c|c|}
\hline Variables & Mean or frequency & SD or percent & Maximum & Minimum \\
\hline Age (year) & 37 & 13 & 69 & 18 \\
\hline Weight (kg) & 72.82 & 16.06 & 138.1 & 41.1 \\
\hline $\mathrm{BMI}\left(\mathrm{kg} / \mathrm{m}^{2}\right)$ & 25.65 & 4.69 & 49.1 & 15.1 \\
\hline LBM (kg) & 47.29 & 11.47 & 89.5 & 28.5 \\
\hline Right arm lean (kg) & 2.69 & 0.91 & 5.88 & 1.1 \\
\hline Left arm lean (kg) & 2.66 & 0.91 & 5.7 & 1.12 \\
\hline SMM (kg) & 27.86 & 7.44 & 54.2 & 15.2 \\
\hline FM (kg) & 22.55 & 9.41 & 67.7 & 1.7 \\
\hline
\end{tabular}


Table I. Characteristics, anthropometric, body composition, and physical fitness measurements (cont.)

\begin{tabular}{|c|c|c|c|c|c|}
\hline \multicolumn{2}{|l|}{ Variables } & Mean or frequency & SD or percent & Maximum & Minimum \\
\hline \multicolumn{2}{|l|}{ PBF } & 30.62 & 9.34 & 53.8 & 3.4 \\
\hline \multicolumn{2}{|c|}{ Visceral fat area $\left(\mathrm{cm}^{2}\right)$} & 104.68 & 48.49 & 262.9 & 5 \\
\hline \multicolumn{2}{|l|}{ Trunk fat (kg) } & 11.36 & 4.65 & 28.4 & 0.1 \\
\hline \multicolumn{2}{|l|}{ WC $(\mathrm{cm})$} & 89.72 & 12.58 & 138.1 & 62.2 \\
\hline \multicolumn{2}{|c|}{ Hip Circumference $(\mathrm{cm})$} & 98.85 & 7.65 & 130.2 & 76.79 \\
\hline \multicolumn{2}{|l|}{ WHC } & 0.9 & 0.07 & 1.12 & 0.77 \\
\hline \multicolumn{2}{|l|}{$\mathrm{BMC}(\mathrm{kg})$} & 2.91 & 0.89 & 12.3 & 1.85 \\
\hline \multicolumn{2}{|l|}{ SBP } & 113.47 & 13.27 & 166 & 85 \\
\hline \multicolumn{2}{|l|}{ DBP } & 71.16 & 9.19 & 102 & 50 \\
\hline \multicolumn{2}{|c|}{ Left handgrip strength (kg) } & 29.69 & 11.54 & 70.4 & 9.1 \\
\hline \multicolumn{2}{|c|}{ Right handgrip strength $(\mathrm{kg})$} & 32.26 & 12.28 & 74.6 & 10.2 \\
\hline \multicolumn{2}{|c|}{ Average handgrip strength $(\mathrm{kg})$} & 33.47 & 12.36 & 72.95 & 12.25 \\
\hline \multicolumn{2}{|c|}{ Average handgrip strength $(\mathrm{kg} / \mathrm{kg} \mathrm{W})$} & 0.46 & 0.13 & 0.85 & 0.16 \\
\hline \multicolumn{2}{|c|}{ Average handgrip strength (kg/kg LBM) } & 0.7 & 0.15 & 1.26 & 0.33 \\
\hline \multicolumn{2}{|c|}{$\mathrm{VO}_{2}$ peak (ml/kg/min) } & 31 & 8 & 54 & 11 \\
\hline \multicolumn{2}{|l|}{$\mathrm{VO}_{2}$ peak $(1 / \mathrm{min})$} & 2.26 & 0.73 & 4.61 & 0.83 \\
\hline \multicolumn{2}{|c|}{$\mathrm{VO}_{2}$ peak (ml/kg LBM/min) } & 47.46 & 8.18 & 76.14 & 22.32 \\
\hline \multirow[t]{3}{*}{ Education status } & Illiterate & 1 & 0.4 & & \\
\hline & Elementary education & 70 & 25.8 & & \\
\hline & College education & 200 & 73.8 & & \\
\hline \multirow[t]{2}{*}{ Marital status } & Single & 125 & 46.5 & & \\
\hline & Married & 144 & 53.5 & & \\
\hline \multirow[t]{2}{*}{ Sex } & Men & 120 & 44.1 & & \\
\hline & Women & 152 & 55.9 & & \\
\hline \multirow{3}{*}{$\begin{array}{l}\text { Physical activity } \\
\text { level }\end{array}$} & Low & 100 & 37.3 & & \\
\hline & Moderate & 113 & 42.2 & & \\
\hline & High & 55 & 20.5 & & \\
\hline \multirow[t]{2}{*}{ Smoking } & Smokers & 36 & 13.3 & & \\
\hline & Non-smokers & 234 & 86.7 & & \\
\hline
\end{tabular}

Data are presented in mean, SD, frequency, percent, minimum and maximum of range.

$\mathrm{BMI}$ - body mass index; LBM - lean body mass; SMM - skeletal muscle mass; FM - fat mass; WC - waist circumference; WHR - waist hip ratio; $\mathrm{BMC}$ - bone mineral content; SBP - systolic blood pressure; DBP - diastolic blood pressure; PBF - percent body fat 
Związek pomiędzy aktywnością fizyczną a wydolnością sercowo-naczyniową,

The participants with higher PAL had significantly the greatest average of LBM ( $p<0.001)$, lean mass of right $(p<0.001)$, and left $(p<0.001)$ arms, skeletal muscle mass (SMM) $(p<0.001)$, and BMC $(p<0.001)$. Furthermore, we observed a significant negative relationship between PAL with FM $(p=0.002)$, percent body fat (PBF) $(p<0.001)$, visceral fat area (VFA) $(p=0.001)$, and trunk fat $(\rho=0.005)$, which demonstrated the lowest amount of fat in high PAL group. No significant associations were found between PAL and weight, body mass index (BMI), and waist-hip ratio (WHR). After adjusting for confounders, a significant association was found between PAL and WHR ( $p=0.02)$. Association between PAL categories and body composition is shown in Table II.

Associations between physical fitness tests and PAL are demonstrated in Table III. The PAL had a direct association with CRF $(p=0.001)$. All indices of muscle strength test significantly increased with enhanced levels of PA $(p<0.001)$. After adjusting for age and gender, results demonstrated a significant association between $\mathrm{PAL}$ and $\mathrm{VO}_{2}$ peak, when expressed in $\mathrm{ml} / \mathrm{min} / \mathrm{kg}$ BW $(p<0.001)$ or $1 / \min (p=0.002)$ and the average of right and left handgrip (and $\mathrm{kg} / \mathrm{kg} \mathrm{BW}[p=0.016]$ ). These relations among PAL with average right and left handgrip strength $(\mathrm{kg}$; $p=0.05,(\mathrm{~kg} / \mathrm{kg} \mathrm{BW} ; p=0.027)$ and $\mathrm{VO}_{2}$ peak $(\mathrm{ml} / \mathrm{min} / \mathrm{kg} \mathrm{BW}$; $p<0.001, \mathrm{l} / \mathrm{min} ; p=0.001$ ) remained significant after adjusting for BMI, age, and gender. Moreover, there was an association between PAL with left handgrip strength $(\mathrm{kg} ; p=0.045)$.

Associations between the continuous score of PA with body composition (LBM, PBF, visceral fat area, BMI) are presented in Figure 1. Right and left handgrip strength $(\mathrm{kg} / \mathrm{kg} \mathrm{W}), \mathrm{VO}_{2}$ peak ( $\mathrm{ml} / \mathrm{kg} / \mathrm{min}$ ), and body composition (except for BMI) were associated with PA.

\section{Discussion}

The results revealed that PAL is related to body composition, muscle strength, and CRF in Iranian adults. However, no significant association was found between PA and weight and BMI. PA also had an association with physical fitness indicators in which higher PA led to better fitness. Additionally, the mean of muscle strength increased across higher levels of PA

In line with our results, Zanovec et al. evaluated PA by IPAQ in their study and reported that there was no significant difference among the mean weight and BMI across PAL categories [15]. This is probably since both weight and BMI are affected by muscle mass and fat mass, and these factors are influenced differently by physical activity. In an investigation on

Table II. The relationship between PAL and body composition measurements in crude and adjusted models

\begin{tabular}{|c|c|c|c|c|c|c|}
\hline \multirow[t]{2}{*}{ Measurements } & \multicolumn{3}{|c|}{ Physical activity levels } & \multirow{2}{*}{$\begin{array}{l}\text { Crude } \\
p\end{array}$} & \multirow{2}{*}{$\begin{array}{l}\text { Model } 1 \\
p\end{array}$} & \multirow{2}{*}{$\begin{array}{l}\text { Model } 2 \\
p\end{array}$} \\
\hline & Low & Moderate & Vigorous & & & \\
\hline Weight (kg) & $73.21(15.86)$ & $70.98(16.49)$ & $76.1(15.68)$ & 0.15 & 0.276 & 0.249 \\
\hline $\mathrm{BMI}\left(\mathrm{kg} / \mathrm{m}^{2}\right)$ & $26.07(4.52)$ & $25.42(5.3)$ & $25.39(3.71)$ & 0.547 & 0.369 & 0.317 \\
\hline LBM (kg) & 45.69 (10.34) & $45.8(10.72)$ & $53.67(13.02)$ & $<0.001$ & 0.009 & 0.01 \\
\hline Right arm lean (kg) & $2.56(0.81)$ & $2.59(0.88)$ & $3.17(1.02)$ & $<0.001$ & 0.013 & 0.014 \\
\hline Left arm lean (kg) & $2.52(0.82)$ & $2.56(0.88)$ & $3.15(1.01)$ & $<0.001$ & 0.008 & 0.008 \\
\hline SMM (kg) & $26.76(6.6)$ & $27(7.16)$ & $31.87(8.32)$ & $<0.001$ & 0.025 & 0.026 \\
\hline FM (kg) & $24.78(9.12)$ & $22.1(9.82)$ & $19.19(8.38)$ & 0.002 & 0.014 & 0.011 \\
\hline PBF & $33.32(8.14)$ & $30.62(9.21)$ & $25.21(9.64)$ & $<0.001$ & 0.001 & $<0.001$ \\
\hline Visceral fat area $\left(\mathrm{cm}^{2}\right)$ & $116.9(46.79)$ & $101.23(49.22)$ & $87.55(45.75)$ & 0.001 & 0.009 & 0.009 \\
\hline Trunk fat (kg) & $12.42(4.48)$ & $11.05(4.77)$ & $9.97(4.48)$ & 0.005 & 0.016 & 0.012 \\
\hline WC (cm) & $91.25(1.3)$ & $88.43(1.19)$ & $89.48(1.62)$ & 0.267 & 0.091 & 0.075 \\
\hline WHR & $0.915(0.07)$ & $0.896(0.06)$ & $0.895(0.07)$ & 0.08 & 0.022 & 0.02 \\
\hline BMC (kg) & $2.8(0.58)$ & $2.79(0.65)$ & $3.4(1.46)$ & $<0.001$ & 0.019 & 0.02 \\
\hline
\end{tabular}

Data are presented in mean (SD). Model 1 was adjusted with age and sex; Model 2 was adjusted with age, sex, and smoking status.

$\mathrm{BMI}$ - body mass index; LBM - lean body mass; SMM - skeletal muscle mass; FM - fat mass; WC - waist circumference; WHR - waist hip ratio; $\mathrm{BMC}$ - bone mineral content; PBF - percent body fat 
Table III. The relationship between PAL and physical fitness tests in crude and adjusted models

\begin{tabular}{lllllll}
\hline \multirow{2}{*}{ Measurements } & \multicolumn{2}{l}{ Physical activity levels } & Crude & Model 1 & Model 2 \\
\cline { 2 - 7 } & Low & Moderate & Vigorous & $p$ & $p$ & $p$ \\
\hline Right handgrip strength $(\mathrm{kg})$ & $30.25(10.93)$ & $31.56(11.79)$ & $38.19(13.82)$ & $<0.001$ & 0.123 & 0.1 \\
\hline Left handgrip strength $(\mathrm{kg})$ & $27.78(10.35)$ & $28.84(11.45)$ & $35.67(12.01)$ & $<0.001$ & 0.057 & 0.045 \\
\hline Average handgrip strength $(\mathrm{kg})$ & $31.34(10.97)$ & $32.68(12.1)$ & $39.82(13.31)$ & $<0.001$ & 0.069 & 0.05 \\
\hline Average handgrip strength $(\mathrm{kg} / \mathrm{kg} \mathrm{W})$ & $0.43(0.12)$ & $0.46(0.13)$ & $0.52(0.14)$ & $<0.001$ & 0.016 & $\mathbf{0 . 0 2 7}$ \\
\hline $\mathrm{VO}_{2}$ peak $(\mathrm{ml} / \mathrm{kg} / \mathrm{min})$ & $29(6)$ & $31(8)$ & $36(9)$ & $<0.001$ & $<0.001$ & $<0.001$ \\
\hline $\mathrm{VO}_{2}$ peak $(\mathrm{l} / \mathrm{min})$ & $2.14(0.63)$ & $2.15(0.71)$ & $2.73(0.79)$ & $<0.001$ & 0.002 & 0.001 \\
\hline $\mathrm{VO}_{2}$ peak $(\mathrm{ml} / \mathrm{kg} \mathrm{LBM} / \mathrm{min})$ & $46.52(7.22)$ & $46.66(7.98)$ & $51.08(9.35)$ & $\mathbf{0 . 0 0 1}$ & 0.14 & 0.142 \\
\hline
\end{tabular}

Data are presented in mean (SD).

Model 1 was adjusted with age and sex; Model 2 was adjusted with age, sex, and BMI.

elementary school students carried out by Cho et al., a higher $\mathrm{BMI}$ and weight were determined among the inactive group. They also observed that inactive participants appeared to have higher FM and PBF which was confirmed by our results [31]. Khona et al. observed a negative correlation between PA and PBF and visceral fat [32]. However, the findings of another study indicated that moderate PA did not lead to a lower fat mass [33]. Moreover, Lee et al. concluded that higher PAL leads to the development of muscle mass [34]. Similarly, in the study by Preto et al. on nursing students, researchers found a positive association between PA and muscle mass [35].

Another important finding was the positive association between PA and muscle strength. In line with our findings, Allie Leblanc et al. found a correlation between PA and HGS In contrast to our results, Gerdhem et al. and Morie et al. have reported no associations between PA and HGS $[36,37]$. The findings of another study showed that PA is related to HGS in adults [38]. Overall, several studies demonstrated that although both muscle mass and strength increased with frequent PA, the development of muscle mass led to the enhancement in strength [39]. Evidence suggests that muscular hypertrophy of both type I and type || muscle fibers occurs with strength training. the increase of muscle mass and muscle strength does not occur in parallel, so that, muscle strength changes were greater than the muscle growth. Past studies revealed that neurological factors and adaptation significantly affect the gain in strength in addition to improvement in muscle morphology [40].

Our study identified a positive association between PA and CRF. In line with our findings, Khona et al. and Fogelholm et al. found that PA had a positive effect on $\operatorname{CRF}[32,41]$. Likewise, Dencker et al. in a study on 8 to 11-year-old children reported a significant relationship between aerobic fitness and PA [42]. In contrast, others found no association between PAL and CRF in adolescent boys [43] and children [44].
This study suggests that PA has a stronger relationship with CRF rather than muscle strength. The processes by which physical activity improve CRF have remained unclear [45]. Cardiac and vascular changes including cardiac hypertrophy, increasing cardiac output, and promotion of oxygen-carrying capacity result from exercise. Maybe, these changes linked to better CRF [46].

Moreover, PA has revealed a stronger relationship with percent body fat in comparison with LBM. Some evidence $[47,48]$ have demonstrated that CRF is strongly related to fat mass, and on the other hand, there is a strong association between muscle strength and muscle mass. It seems that habitual PA generates a greater impact on fat mass than muscle mass and as a result a more intense effect on CRF [49]. Moreover, this may suggest that intense and durable (last long, enduring, continual) activities lead to muscle mass growth, in addition to decreasing fat mass, and in turn, this will result in the improvement of muscle strength.

This study was performed among both genders and a wide age range, which led to their confounding effects being eliminated. The limitations of this study included its cross-sectional and observational design. Also, IPAQ does not assess activities that are done in less than 10 minutes. But the inclusion of activities related to different domains of life, not just the leisure-time physical activity, improves the accuracy of evaluation.

\section{Conclusions}

Our findings suggest that promoting intensity of PA leads to a better body composition profile, physical fitness, and eventually health promotion. However, PAL could be a reliable predictor of body composition, muscular and cardiovascular fitness status. Further longitudinal studies are warranted to understand the causal direction of the relationship. 

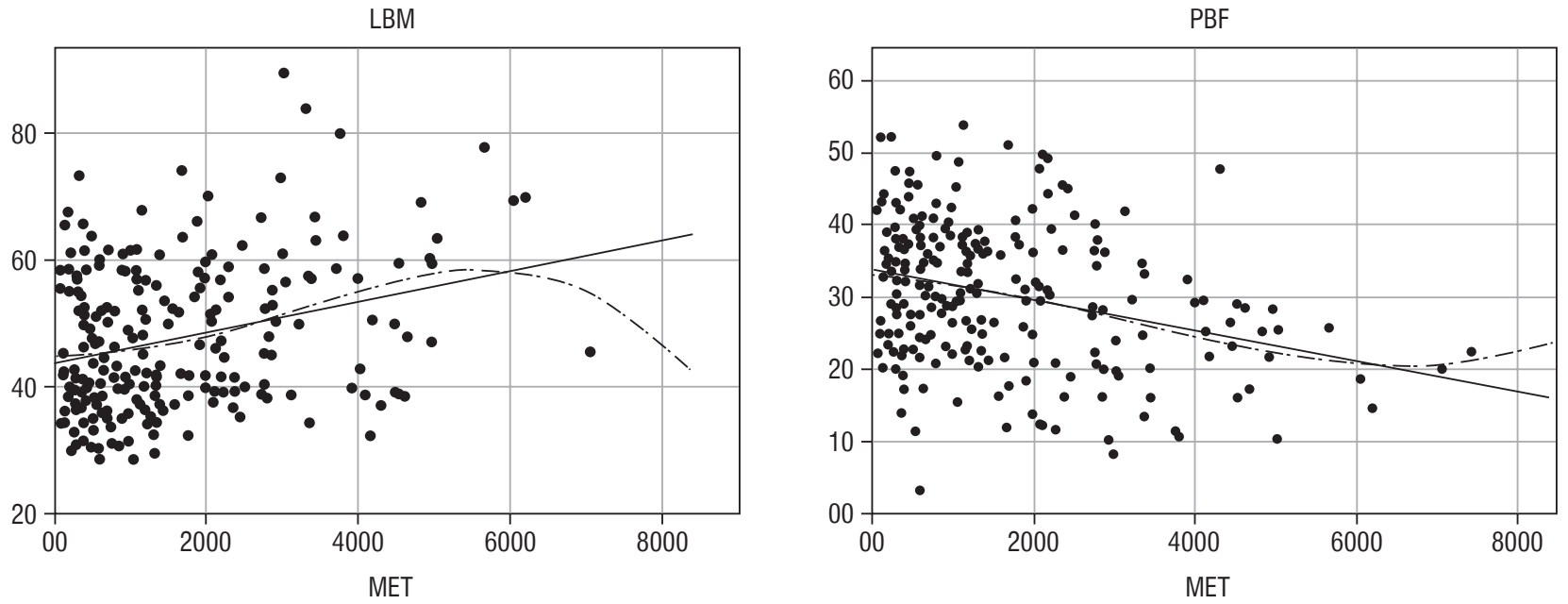

- Observed — Linear -- Cubic $\beta=0.296 \quad p<0.001$

- Observed — Linear -.-. Cubic $\beta=0.317 \quad p<0.001$

VFA
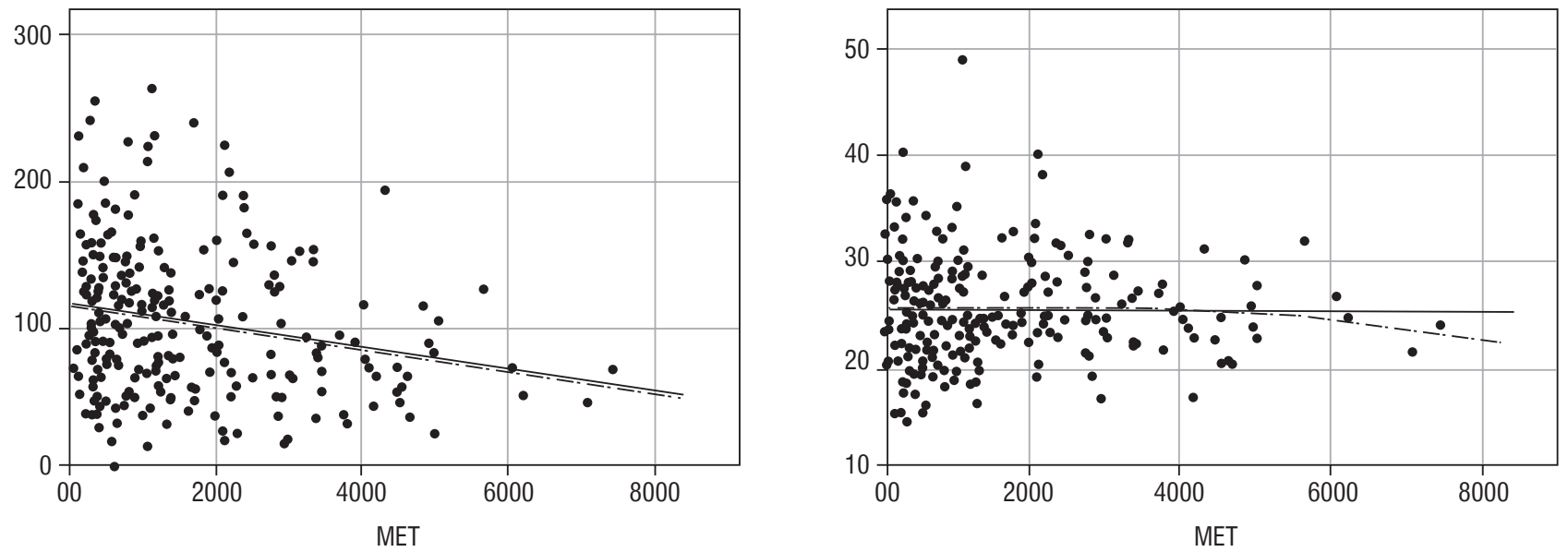

- Observed —- Linear -.- Cubic $\beta=0.214 \quad p<0.001$

- Observed L Linear -.-- Cubic $\quad \beta=0.014 \quad p=0.815$

$\mathrm{VO}_{2}$ peak

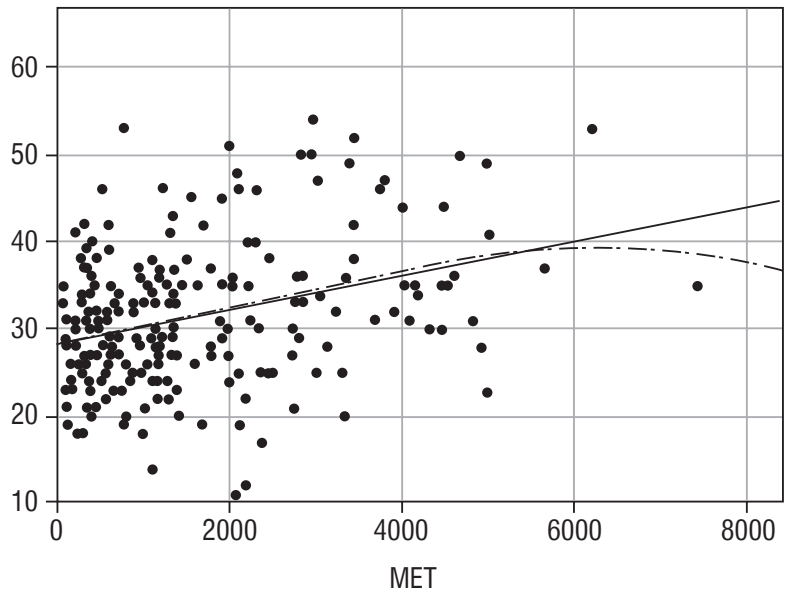

- Observed —Linear -- Cubic $\beta=0.355 \quad p<0.001$

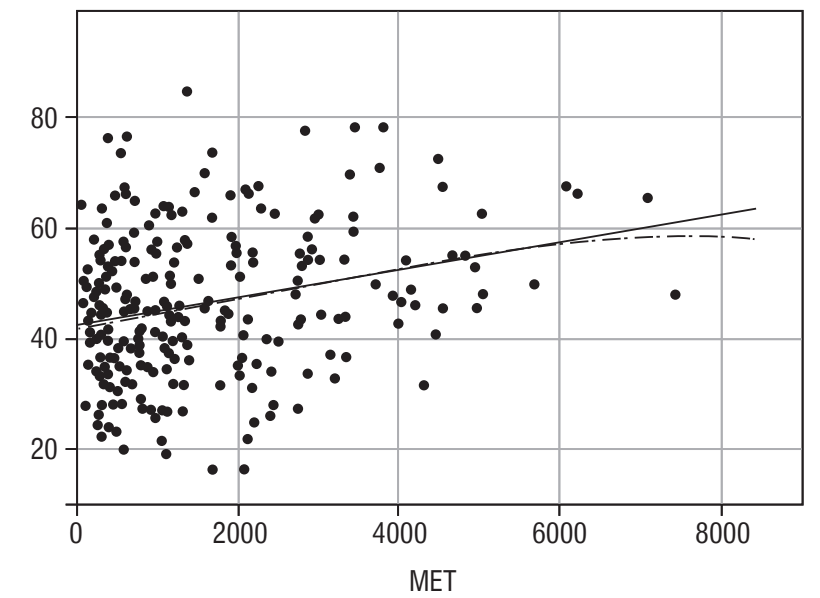

- Observed L- Linear -- Cubic $\quad \beta=0.266 \quad p<0.001$

Figure 1. Associations between continuous score of PA with some selected factors of body composition 


\section{References}

1. Organization WH. Global recommendations on physical activity for health. WHO, Geneva 2010; 1-53.

2. Haskell WL, Lee IM, Pate RR, et al. Physical activity and public health: updated recommendation for adults from the American College of Sports Medicine and the American Heart Association. Med Sci Sports Exerc 2007; 39: 1423-1434. doi: 10.1249/mss.0b013e3180616b27

3. Warburton DE, Nicol CW, Bredin SS. Health benefits of physical activity: the evidence. CMAJ 2006; 174: 801-809. doi: 10.1503/ cmaj.051351

4. Monyeki $\mathrm{M}$, Kemper $\mathrm{H}$. Is there a positive relationship between physical fitness and physical activity in children? A brief review. J Exercise Sci Physiother 2013; 3: 12-16.

5. Morrow JR Jr., Tucker JS, Jackson AW, et al. Meeting physical activity guidelines and health-related fitness in youth. Am J Prev Med 2013; 44: 439-444. doi: 10.1016/j.amepre.2013.01.008

6. Janssen I, Leblanc AG. Systematic review of the health benefits of physical activity and fitness in school-aged children and youth. Int J Behav Nutr Phys Act 2010; 7: 40.

7. Molnar D, Livingstone B. Physical activity in relation to overweight and obesity in children and adolescents. Eur J Pediatr 2000; 159 Suppl 1: S45-S55. doi: 10.1007/pl00014365

8. Cooper R, Kuh D, Hardy R. Objectively measured physical capability levels and mortality: systematic review and meta-analysis. BMJ 2010; 341: c4467. doi: 10.1136/bmj.c4467

9. Leong DP, Teo KK, Rangarajan S, et al. Prognostic value of grip strength: findings from the Prospective Urban Rural Epidemiology (PURE) study. Lancet 2015; 386: 266-273. doi: 10.1016/S01406736(14)62000-6

10. Lima LP, Leite HR, Matos MA, et al. Cardiorespiratory fitness assessment and prediction of peak oxygen consumption by Incremental Shuttle Walking Test in healthy women. PLoS One 2019; 14 : e0211327. doi: 10.1371/journal.pone.0211327

11. de Zwart AH, Dekker J, Lems W, et al. Factors associated with upper leg muscle strength in knee osteoarthritis: A scoping review. J Rehabil Med 2018; 50: 140-150. doi: 10.2340/16501977-2284

12. Massy-Westropp NM, Gill TK, Taylor AW, et al. Hand Grip Strength: age and gender stratified normative data in a population-based study. BMC Res Notes 2011; 4: 127. doi: 10.1186/1756-0500-4-127

13. Norman K, Stobäus N, Gonzalez MC, et al. Hand grip strength: outcome predictor and marker of nutritional status. Clin Nutr 2011; 30: 135-142. doi: 10.1016/j.clnu.2010.09.010

14. Blair SN, Church TS. The fitness, obesity, and health equation: is physical activity the common denominator? JAMA 2004; 292: 1232-1234. doi: 10.1001/jama.292.10.1232

15. Zanovec M, Lakkakula AP, Johnson LG, et al. Physical Activity is Associated with Percent Body Fat and Body Composition but not Body Mass Index in White and Black College Students. Int J Exerc Sci 2009; 2: 175-185.

16. Burgi $F$, Meyer $U$, Granacher $U$, et al. Relationship of physical activity with motor skills, aerobic fitness and body fat in preschool children: a cross-sectional and longitudinal study (Ballabeina). Int J Obes (Lond) 2011; 35: 937-944. doi: 10.1038/ijo.2011.54

17. Leppanen MH, Nystrom CD, Henriksson P, et al. Physical activity intensity, sedentary behavior, body composition and physical fitness in 4-year-old children: results from the ministop trial. Int $\mathrm{J}$ Obes (Lond) 2016; 40: 1126-1133. doi: 10.1159/000097979

18. Forrest KY, Zmuda JM, Cauley JA. Patterns and correlates of muscle strength loss in older women. Gerontology 2007; 53: 140-147. doi: 10.1159/000097979

19. Church TS, Earnest CP, Skinner JS, et al. Effects of different doses of physical activity on cardiorespiratory fitness among sedentary, overweight or obese postmenopausal women with elevated blood pressure: a randomized controlled trial. JAMA 2007; 297: 2081-2091. doi: 10.1001/jama.297.19.2081

20. Dencker M, Andersen LB. Health-related aspects of objectively measured daily physical activity in children. Clin Physiol Funct Imaging 2008; 28: 133-144. doi: 10.1111/j.1475-097X.2008.00788.x

21. Huang YC, Malina RM. Physical activity and health-related physical fitness in Taiwanese adolescents. J Physiol Anthropol Appl Human Sci 2002; 21: 11-19. doi: 10.2114/jpa.21.11

22. Matton L, Thomis M, Wijndaele $\mathrm{K}$, et al. Tracking of physical fitness and physical activity from youth to adulthood in females. Med Sci Sports Exerc 2006; 38: 1114-1120. doi: 10.1249/01. mss.0000222840.58767.40

23. Malina RM. Physical activity and fitness: pathways from childhood to adulthood. Am J Hum Biol 2001; 13: 162-172. doi: 10.1002/1520-6300(200102/03)13:2<162::AID-AJHB1025> 3.0.CO;2-T

24. Maggio AB, Hofer MF, Martin XE, et al. Reduced physical activity level and cardiorespiratory fitness in children with chronic diseases. Eur J Pediatr 2010; 169: 1187-1193. doi: 10.1007/s00431010-1199-2

25. Zanovec M, Lakkakula AP, Johnson LG, et al. Physical activity is associated with percent body fat and body composition but not body mass index in white and black college students. Int $\mathrm{J}$ Exerc Sci 2009; 2: 175-185.

26. Schiavo L, Scalera G, Pilone V, et al. Fat mass, fat-free mass, and resting metabolic rate in weight-stable sleeve gastrectomy patients compared with weight-stable nonoperated patients. Surg Obes Relat Dis 2017; 13: 1692-1699. doi: 10.1016/j.soard.2017.06.007

27. Reis MM, Arantes PMM. Assessment of hand grip strength-validity and reliability of the saehan dynamometer. Fisioter Pesqui 2011; 18 : 176-181. doi: https://doi.org/10.1590/S1809-29502011000200013

28. Hamlin M, Draper N, Blackwell G, et al. Determination of maximal oxygen uptake using the bruce or a novel athlete-led protocol in a mixed population. J Hum Kinet 2012; 31: 97-104. doi: 10.2478/ v10078-012-0010-z

29. Committee IR. Guidelines for data processing and analysis of the International Physical Activity Questionnaire (IPAQ)-short and long forms. http://www.ipaq.ki.se/scoring.pdf.2005.

30. Moghaddam MB, Aghdam FB, Jafarabadi MA, et al. The Iranian Version of International Physical Activity Questionnaire (IPAQ) in Iran: content and construct validity, factor structure, internal consistency and stability. World Appl Sci J 2012; 18: 1073-1080. doi: 10.5829/idosi.wasj

31. Cho M, Kim J-Y. Changes in physical fitness and body composition according to the physical activities of Korean adolescents. J Exerc Rehabil 2017; 13: 568-572. doi: 10.12965/jer.1735132.566.

32. Khona NN, Maiya AG, Acharya K, et al. Correlation of physical activity level with bone mineral density, cardio-respiratory fitness and 
body composition in post-menopausal women. Int $\mathrm{J}$ Physiother 2017; 4: 6-11. doi: 10.15621/ijphy/2017/v4i1/136154

33. Sims ST, Larson JC, Lamonte MJ, et al. Physical activity and body mass: changes in younger versus older postmenopausal women. Med Sci Sports Exerc 2012; 44: 89-97. doi: 10.1249/MSS. Ob013e318227f906

34. Lee KS, Lee JK, Yeun YR. Effects of a 10-day intensive health promotion program combining diet and physical activity on body composition, physical fitness, and blood factors of young adults: a randomized pilot study. Med Sci Monit 2017; 23: 1759-1767. doi: $10.12659 / \mathrm{msm} .900515$

35. Preto L, Novo A, Mendes M. Relationship between physical activity, muscle strength and body composition in a sample of nursing students. Rev Enf Ref 2016; 4: 81-89.

36. Gerdhem P, Dencker M, Ringsberg K, et al. Accelerometer-measured daily physical activity among octogenerians: results and associations to other indices of physical performance and bone density. Eur J Appl Physiol 2008; 102: 173-180. doi: 10.1007/ s00421-007-0571-z

37. Morie M, Reid KF, Miciek R, et al. Habitual physical activity levels are associated with performance in measures of physical function and mobility in older men. J Am Geriatr Soc 2010; 58: 1727-1733. doi: 10.1111/j.1532-5415.2010.03012.x

38. Rojer AG, Reijnierse EM, Trappenburg MC, et al. Instrumented assessment of physical activity is associated with muscle function but not with muscle mass in a general population. J Aging Health 2018 30: 1462-1481. doi: 10.1177/0898264317721554

39. Goodpaster BH, Chomentowski P, Ward BK, et al. Effects of physical activity on strength and skeletal muscle fat infiltration in older adults: a randomized controlled trial. J Appl Physiol (1985) 2008; 105: 1498-1503. doi: 10.1152/japplphysiol.90425.2008
40. Hoffman J. Physiological aspects of sport training and performance. Human Kinetics 2014

41. Fogelholm M, Malmberg J, Suni J, et al. International physical activity questionnaire: validity against fitness. Med Sci Sports Exerc 2006; 38: 753-760. doi: 10.1249/01.mss.0000194075

42. Dencker M, Thorsson O, Karlsson MK, et al. Daily physical activity and its relation to aerobic fitness in children aged $8-11$ years. Eur J Appl Physiol 2006; 96: 587-592. doi: 10.1007/s00421-005-0117-1

43. Ekelund U, Poortvliet E, Nilsson A, et al. Physical activity in relation to aerobic fitness and body fat in 14- to 15-year-old boys and girls. Eur J Appl Physiol 2001; 85: 195-201. doi: 10.1007/s004210100460

44. Blaes A, Baquet $G$, Fabre $C$, et al. Is there any relationship between physical activity level and patterns, and physical performance in children? Int J Behav Nutr Phys Act 2011; 8: 122. doi: 10.1186/1479-5868-8-122

45. Nystoriak MA, Bhatnagar AJ. Cardiovascular effects and benefits of exercise. Front Cardiovasc Med 2018; 5: 135. doi: 10.3389/ fcrm.2018.00135

46. Vega RB, Konhilas JP, Kelly DP, et al. Molecular mechanisms underlying cardiac adaptation to exercise. 2017; 25: 1012-1026. doi: 10.1016/j.cmet.2017.04.025

47. Goran M, Fields D, Hunter G, et al. Total body fat does not influence maximal aerobic capacity. Int J Obes (Lond) 2000; 24: 841-848. doi: 10.1038/sj.ijo.0801241

48. Ylihärsilä $H$, Kajantie $E$, Osmond $C$, et al. Birth size, adult body composition and muscle strength in later life. Int $\mathrm{J}$ Obes (Lond) 2007; 31: 1392-1399. doi: 10.1038/sj.ijo.0803612

49. Leblanc A, Taylor BA, Thompson PD, et al. Relationships between physical activity and muscular strength among healthy adults across the lifespan. Springerplus 2015; 4: 557. doi: 10.1186/ s40064-015-1357-0 